\title{
Electron correlations in the $L$-shell photoionization of heavy elements
}

\author{
W. Jitschin* \\ Physikalisch-Technische Bundesanstalt, Institut Berlin, D-1000 Berlin 10, Germany \\ U. Werner \\ Fakultät für Physik, Universität Bielefeld, D-4800 Bielefeld 1, Germany \\ G. Materlik \\ Hamburger Synchrotronstrahlungslabor (HASYLAB), Deutsches Elektronen-Synchrotron (DESY), \\ D-2000 Hamburg 52, Germany \\ G. D. Doolen \\ Los Alamos National Laboratory, Los Alamos, New Mexico 87545
}

(Received 4 August 1986)

\begin{abstract}
The photoionization of the individual $L$ subshells of ${ }_{72} \mathrm{Hf},{ }_{74} \mathrm{~W},{ }_{78} \mathrm{Pt},{ }_{79} \mathrm{Au}$, and ${ }_{82} \mathrm{~Pb}$ in the energy regime of the $L$ edges has been studied in detail. Experimentally, the x-ray absorption spectra of thin sample foils were recorded using monochromatized synchrotron radiation. The energy dependence of the absorption is governed in its gross structure by the atomic photoionization with some superimposed oscillatory structure due to solid-state effects. The experimental data are compared to various theoretical predictions for atomic photoionization. Calculations in the framework of an independent-electron approach predict a smooth, power-law-like energy dependence. In contrast, the experimental data show small but significant deviations from such a behavior. The dispersionlike deviations are attributed to electron-correlation effects, as is confirmed by comparative calculations of photoionization with inclusion or omission of the correlations using the computer code of Liberman and Zangwill [Comput. Phys. Commun. 32, 75 (1984)]. The main influence of the correlation effects on the subshell ionization cross sections originates from the dielectric (anti-) screening of the external radiation field. Calculations in the local-density approximation for the response of the atom to the radiation field yield an even quantitative description of the experimental data in a large fraction of the investigated energy range.
\end{abstract}

\section{INTRODUCTION}

Inner-shell processes have attracted much attention in recent years. ${ }^{1}$ On the one hand, these processes are of fundamental simplicity. For example, ionization by photons as well as by electron and light-ion impact may be treated in a perturbative approach and model calculations assuming independent inner-shell electrons generally yield reasonable predictions. On the other hand, advanced experiments have revealed small characteristic deviations from the predictions. A detailed study of these deviations may play a key role for understanding subtle effects, as, e.g., resulting from the projectile deflection or the collision-induced perturbation of atomic eigenstates. ${ }^{2-5}$ Photoionization studies are preferable to investigate the response of an atom to an external perturbation: Theoretically, the photon-atom interaction is very weak at common intensities making a perturbative approach well justified; experimentally, photoionization cross sections can be obtained with comparatively high accuracy. Cross sections can be determined directly from the measured x-ray mass attenuation and one need not worry about uncertain fluorescence or Auger yields in evaluating emission spectra. The availability of monochromatized, tunable synchrotron radiation makes advanced photoionization exper- iments feasible. Most of the recent work has concentrated on outer and intermediate shells where a failure of the independent-electron approach is frequently observed (see, e.g., Refs. 6-9): Experimental cross sections deviate from theoretical predictions in magnitude and may exhibit significant unexpected structure; also one-photon-twoelectron transitions may take place.

For inner-electron shells one expects electron correlations to be small on account of the dominating Coulomb potential of the nucleus. A very sensitive probe for electron-correlation effects is the nonradiative vacancy decay which per se is a two-electron process. ${ }^{10,11}$ Theoretical models have to treat at least two active electrons; nevertheless, calculated Coster-Kronig rates and concomitant level widths can be too large by factors of 2 or 3, as compared to experiment (see, e.g., Refs. 12-14). Photoionization is usually a one-electron process and calculations of inner-shell cross sections in the independentparticle model give reasonable results. For example, Scofield's ${ }^{15}$ calculations of the total photoelectric cross section agree with experiment within a few per cent on an absolute scale for $\mathrm{x}$-ray energies above $10 \mathrm{keV} .{ }^{16}$ The energy spectra of absorption cross sections have been studied so far only in a few cases with high resolution, although monochromatized tunable synchrotron radiation provides 
a powerful tool for corresponding measurements. ${ }^{17}$ In the absorption spectrum of $\mathrm{Ar}$ above the $K$ edge, a slight enhancement of the cross section in the vicinity of the edge, as well as some structure amounting to a few per cent of the total cross section, has been observed. ${ }^{18,19}$ The enhancement results from the dynamical rearrangement of electron shells due to the vacancy creation and decay, ${ }^{8}$ whereas the structure originates from one-photon-twoelectron excitations causing resonancelike profiles at the corresponding thresholds. ${ }^{19}$

In a current research program on the decay of innershell vacancies related to the accurate determination of fluorescence, Auger, and Coster-Kronig rates, we have carefully investigated the vacancy creation by photoionization. The measured absorption spectra reveal small but significant deviations from a smooth energy dependence by a power law with almost constant exponent in the whole energy regime of the $L$ edges as, e.g., predicted by the empirical Victoreen formula. ${ }^{20-22}$ These deviations hamper a separation of the measured total cross section into the contributions from the individual shells and subshells. ${ }^{13}$ Our recent comprehensive experimental data clearly reveal a dispersionlike behavior of the deviations which is a typical signature of electron correlations. This finding is corroborated by comparative calculations in the independent-particle model and in the linear-response approximation to the many-body problem. The present paper reports on this work.

\section{EXPERIMENTAL PROCEDURE}

The x-ray absorption of self-supporting sample foils has been measured in a transmission experiment. The primary $\mathrm{x}$-ray beam was monochromatized, synchrotron radiation available at the Röntgen-monochromator (RÖMO) station of the DORIS II electron synchrotron in Hamburg or at the wiggler beamline of the Synchrotron Radiation Source in Daresbury. At both facilities a double-crystal monochromator in nondispersive arrangement was used; harmonics were suppressed by a slight disalignment of the second crystal with respect to the first one. ${ }^{23}$ The spectral bandwidth and the step width in the scans amounted to several electron volts i.e., smaller than or comparable to the natural widths of all $L$ levels. ${ }^{24}$ Thus, all structures in the absorption spectra are resolved, although there is some instrumental influence on the shape of the sharp peaks near the edges. The samples used (Table I) were commercial high-purity foils supplied by the Goodfellow Company. No significant impurities were observed in the induced x-ray fluorescence spectra. In all measurements the foils were kept at room temperature.

In a test phase of the experiment the $\mathrm{x}$-ray absorption of the individual foils was measured by scanning the primary energy and recording the mass attenuation spectra (Fig. 1). For this purpose the $\mathrm{x}$-ray intensities behind the in front of the absorber foil were recorded by ionization chambers $^{25}$ and the absorption was derived from the in-
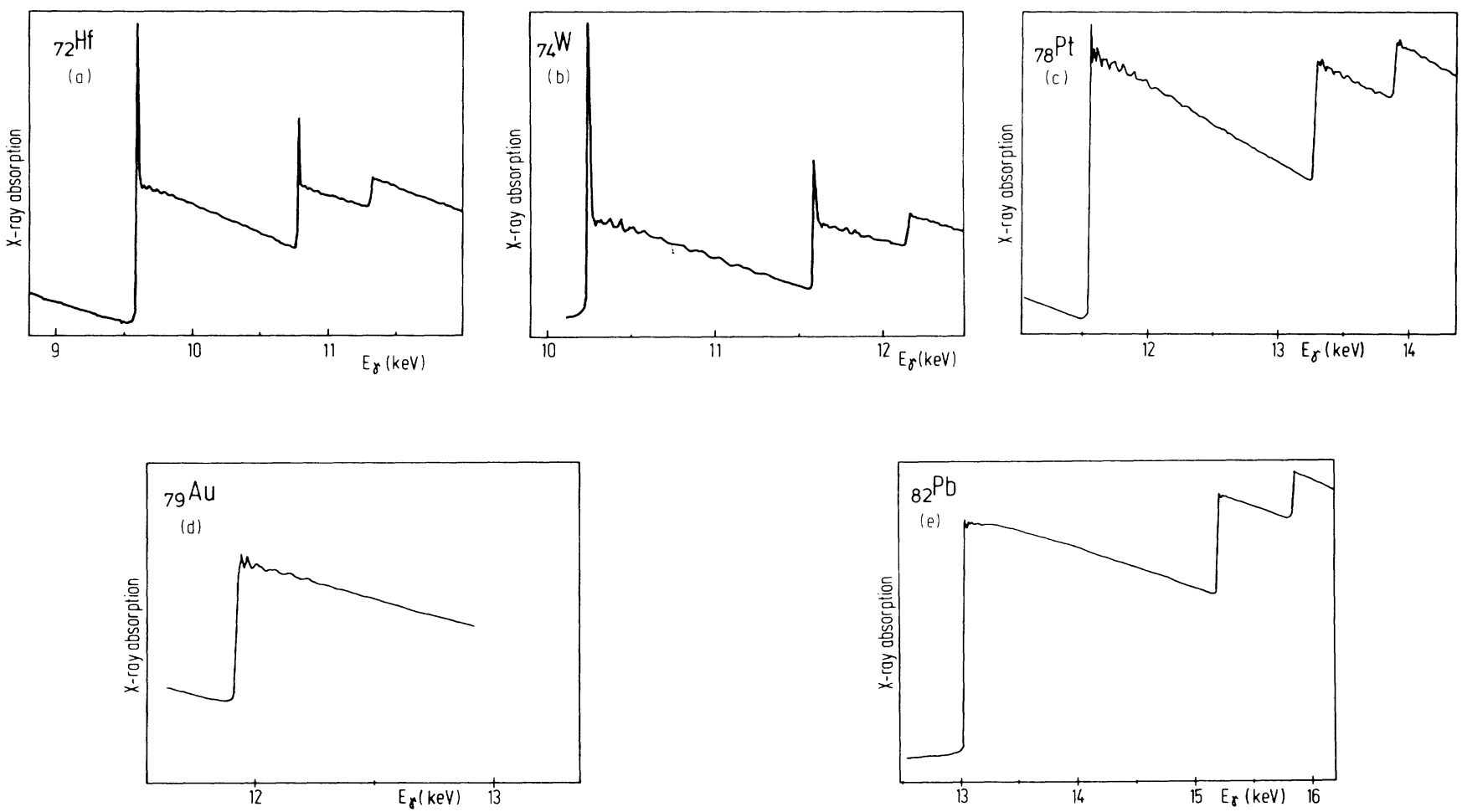

FIG. 1. Scans of the $\mathrm{x}$-ray absorption spectra of (a) ${ }_{72} \mathrm{Hf}$, and (b) ${ }_{74} \mathrm{~W}$ (taken at Hamburg) and (c) ${ }_{78} \mathrm{Pt}$, (d) ${ }_{79} \mathrm{Au}$, and (e) ${ }_{82} \mathrm{~Pb}(\mathrm{taken}$ at Daresbury) recorded at room temperature. Note that the absorption is plotted in arbitrary units which may also vary smoothly with primary energy. 
TABLE I. Sample foils used in the present experiment and their $x$-ray transmission immediately above the $L_{1}$ edge (minimum transmission if overshoot at the white lines is neglected), and immediately below the $L_{3}$ edge (maximum transmission).

\begin{tabular}{|c|c|c|c|}
\hline Sample & $\begin{array}{l}\text { Foil thickness } \\
\qquad(\mu \mathrm{m})\end{array}$ & $\begin{array}{l}\text { Minimum } \\
\text { transmission }\end{array}$ & $\begin{array}{c}\text { Maximum } \\
\text { transmission }\end{array}$ \\
\hline${ }_{72} \mathrm{Hf}$ & 5 & 0.18 & 0.52 \\
\hline${ }_{74} \mathrm{~W}$ & 6 & 0.07 & 0.35 \\
\hline${ }_{78} \mathrm{Pt}$ & 4 & 0.17 & 0.49 \\
\hline${ }_{79} \mathrm{Au}$ & 10 & 0.03 & 0.24 \\
\hline${ }_{82} \mathrm{~Pb}$ & 5 & 0.42 & 0.69 \\
\hline
\end{tabular}

tensity ratio. Since the efficiencies of the two ionization chambers were not equal and different in energy dependence, an additional scan of the primary energy but without absorber was always made. Using the second scan for normalization, the $\mathrm{x}$-ray absorption of the sample foil was determined absolutely. Spectra of the same element taken at Hamburg and at Daresbury looked quite alike as expected.

The absorption spectrum of $\mathrm{Hf}$ shows a strong overshoot ("white line") near the $L_{3}$ and $L_{2}$ edges which originates from photoexcitation from the $L_{3}$ and $L_{2}$ levels to the unfilled $5 d$ level. The overshoot becomes smaller for the heavier elements and finally disappears for $\mathrm{Au}$, i.e., when the $5 d$ level is filled. The oscillatory structure of the cross section above the edges [extended x-ray absorption fine structure (EXAFS)] results from the solidstate effect: reflection of the photoelectron by neighboring atoms (see, e.g., Ref. 26). This EXAFS has already been analyzed in detail for Au (Ref. 27) and for Pt (Ref. 28). For $\mathrm{Pb}$ almost no oscillations are observed presumably due to the low Debye temperature of $\mathrm{Pb}(90 \mathrm{~K})$ which is the lowest of all investigated elements. ${ }^{29}$ In the following we disregard the oscillations since we are interested in atomic properties.

The absorption spectra taken in the test phase turned out to be nicely reproducible in fine details, e.g, the EXAFS oscillations. However, over a broader energy regime (some $100 \mathrm{eV}$ ) they were not reproducible as can already be seen from the different gross shapes of the spectra in Fig. 1. We attribute this nonreproducibility to instabilities and drifts of the apparatus during the long time interval of about $1 \mathrm{~h}$ between the same energy setting in a set of the two scans with and without absorber due to the low scan speed. Reproducible and reliable data were obtained in the final phase of the experiment when a simple method of data taking was used: At fixed primary energy the $x$-ray intensity was measured with the second ionization chamber only, at which time the absorber foil was inserted into or retracted from the $\mathrm{x}$-ray beam. These two intensity measurements could be performed within a few seconds. Data by this method have been taken only at DORIS II in Hamburg.

The absorption was measured at different $\mathrm{x}$-ray energies which were chosen uncorrelated to the EXAFS oscillations (Fig. 2). Thus changes of the absorption cross section by EXAFS are averaged out in the gross energy dependence and do not disturb the data interpretation. A further point of care is the uniformity of the sample thickness; a nonuniform thickness would result in erroneous smaller absorption data, especially in case of strong $\mathrm{x}$-ray attenuation. We do not regard this problem as serious for our rather thin foils which had only a few tiny holes and modest $\mathrm{x}$-ray absorption (Table I). No attempt was made in our experiment to measure the mass attenuation absolutely as this requires the formidable task of precisely determining the sample thickness. Absolute attenuation values are difficult to measure; severe discrepancies between published values have recently stimulated the Commission on Crystallographic Apparatus of the International Union of Crystallography to institute a project which aims at establishing reliable absolute $\mathrm{x}$-ray attenuation coefficients. ${ }^{30,31}$

\section{COMPARISON OF EXPERIMENT AND INDEPENDENT-PARTICLE THEORIES}

The experimentally measured total $x$-ray attenuation results from various contributing processes: photoionization of all subshells which can be ionized and coherent and incoherent photon scattering. For the systems studied, photoionization is the dominating process, whereas scattering amounts to only a few percent of the total cross section. In order to compare the experimental total $x$-ray attenuation with theory, we add up calculated scattering cross sections $^{32,33}$ and photoionization cross sections. The latter ones will now be discussed in detail.

Photoionization can be calculated by standard theory, assuming hydrogenlike wave functions and dipole interaction between photon and electron. ${ }^{34,35}$ The dependence of the ionization cross section $\sigma_{i}$ of a particular subshell $i$ on the photon energy $E_{\gamma}$ within some energy range shows approximately an exponential behavior:

$$
\sigma_{i} \propto E_{\gamma}^{-n},
$$

where the exponent $n$ depends on the subshell; for the $L$ subshells near their thresholds, we find

$$
\begin{aligned}
& n\left(L_{1}\right)=\frac{25}{12} \approx 2.083, \\
& n\left(L_{2}\right)=\frac{101}{33} \approx 3.061, \\
& n\left(L_{3}\right)=n\left(L_{2}\right) .
\end{aligned}
$$

To apply this theory to inner shells of heavy atoms, a screened nuclear charge may be employed. ${ }^{34}$ Our corresponding calculations were found to agree with advanced calculations $^{15}$ (see below) at least within $30 \%$ absolutely for all $L$ subshells in the energy range of our experiment. This seems a very reasonable agreement considering the simplicity of the model.

One essential shortcoming of this simple approach is the use of hydrogenic wave functions. Advanced calculations by Scofield ${ }^{15}$ overcome this shortcoming and incorporate also the following further refinements. ${ }^{15}$

Electrons move in a Hartree-Slater central potential. This potential is assumed to be the same before and after the absorption of the photon. 

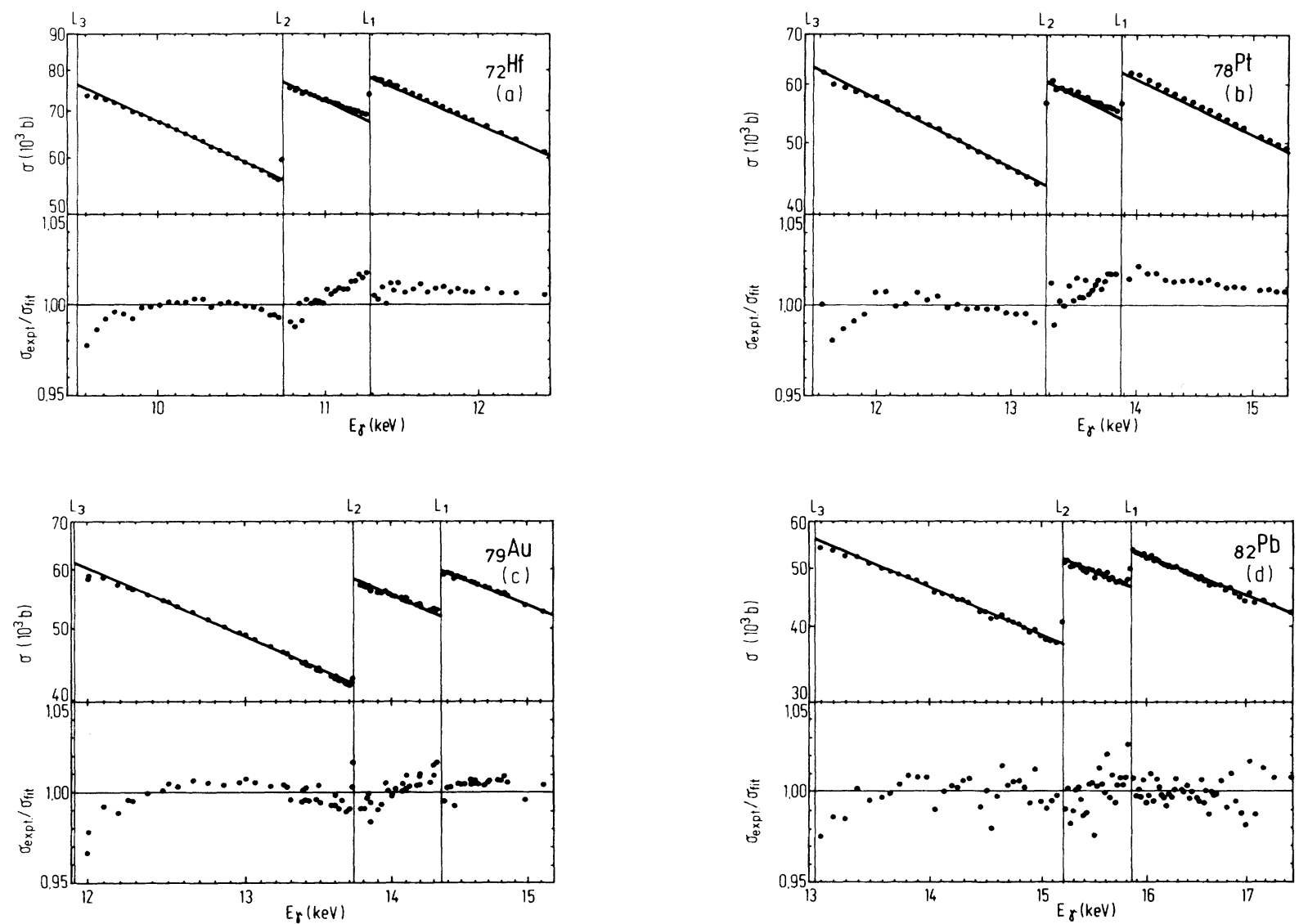

FIG. 2. Quantitative x-ray absorption spectra of $(a){ }_{72} \mathrm{Hf},(\mathrm{b}){ }_{78} \mathrm{Pt},(\mathrm{c}){ }_{79} \mathrm{Au}$, and (d) ${ }_{82} \mathrm{~Pb}$. Upper parts: experimental data all taken at Hamburg and theoretical curves [Eq. (4)]; the relative experimental data have been normalized to theory in the energy regime between the $L_{3}$ and $L_{2}$ edges. Lower part: ratio of experimental data and $\sigma_{\text {total }}^{\text {fit }}$ [Eq. (4)].

Electrons are treated relativistically.

In the treatment of the radiation field all contributing multipoles and retardation effects are included.

Scofield judges his calculations: ${ }^{15}$ "Within the framework of a potential model for the treatment of the electrons, the relativistic calculations represent highly accurate descriptions of the photoionization process. However, there is a crucial question of a proper potential to be used and of the validity of any potential model." These relativistic Hartree-Slater (RHS) calculations have been proved to give a reasonable description of the experimental data: For the systems studied in our experiment, the agreement between RHS prediction and experiment is within 3-5\% absolutely for the total photoelectric cross section. ${ }^{16}$

We now turn to partial cross sections. In an independent-particle approach the cross sections show a smooth energy dependence, since, e.g., for photoionization, the wave function of the final state, i.e., of the photoelectron, changes smoothly with its energy. Theoretical cross sections have been tabulated only for selected primary energies making interpolation necessary. For convenience, we fitted available values by a power-law ansatz which separates the dependencies on atomic number $Z$ and photon energy $E_{\gamma}$ :

$$
\frac{\sigma_{i}\left(Z, E_{\gamma}\right)}{\operatorname{barn}}=a_{i}\left(\frac{Z}{77}\right)^{b_{i}}\left(\frac{E_{\gamma}}{10 \mathrm{keV}}\right)^{c_{i}} \theta_{i},
$$

where $i$ denotes the process and the fit parameters $a_{i}, b_{i}$, and $c_{i}$ are given in Table II. $\theta_{i}$ denotes a switching function which takes the values $\theta_{i}=0$ for primary energies $E_{\gamma}$ smaller than the threshold energy of the process $i$, and $\theta_{i}=1$ elsewhere. The fitted cross sections reproduce the original cross sections with deviations generally well below $1 \%$ and always better than $2 \%$. The remaining deviations are partially due to the crudeness of the ansatz but also partially due to scattering (at most, 1\%) of the original cross sections tabulated by Scofield, ${ }^{15}$ as can also be seen from Fig. 3. The scattering is presumably due to numerical inaccuracies; recent more comprehensive calculations by Scofield ${ }^{36}$ for $\mathrm{Au}$ do not show this scattering. The total absorption cross section is just the sum over all contributions:

$$
\sigma_{\text {total }}^{\mathrm{fit}}\left(Z, E_{\gamma}\right)=\sum_{i=1}^{6} \sigma_{i}^{\mathrm{fit}}\left(Z, E_{\gamma}\right)
$$

Comparison of our experimental data with theoretical pre- 
TABLE II. Coefficients from a fit to tabulated cross sections ${ }^{15,32}$ employing a power-law ansatz [Eq. (3)].

\begin{tabular}{llrcc}
\hline \hline Index $i$ & \multicolumn{1}{c}{ Process } & $a_{i}$ & $b_{i}$ & $c_{i}$ \\
\hline 1 & $L_{1}$ photoionization & 14306 & 1.54 & -1.735 \\
2 & $L_{2}$ photoionization & 32720 & 3.88 & -2.544 \\
3 & $L_{3}$ photoionization & 53678 & 3.94 & -2.752 \\
4 & $M, N, \ldots$ photoionization & 33229 & 4.16 & -2.616 \\
5 & coherent scattering & 1443 & 2.26 & -1.003 \\
6 & incoherent scattering & 15 & 0.55 & +0.680 \\
\hline
\end{tabular}

dictions cannot be made on an absolute scale since our data are not absolute. We arbitrarily decided to normalize our data by matching experiment and theory in the energy regime between the $L_{3}$ and $L_{2}$ edges; the region from the $L_{3}$ edge up to approximately $300 \mathrm{eV}$ immediately above the $L_{3}$ edge was omitted in the normalization in order to avoid disturbances by the near-threshold multielectron effects $^{37}$ and EXAFS oscillations. After the normalization, comparison of our experimental data and the theoretical cross section given by Eq. (4) yields reasonable agreement within $\pm 2 \%$ over the whole investigated energy range. Nevertheless, the following small but significant deviations are observed which are pronounced in a magnified display (Fig. 2, lower parts).
In the energy regime between the $L_{3}$ - and $L_{2}$ edges the experimental data exhibit a curvature, i.e., the slope of the cross section changes considerably with $\mathrm{x}$-ray energy.

Between the $L_{2}$ and $L_{1}$ edges the experimental data exhibit a quite different slope compared to the regime between the $L_{3}$ and $L_{2}$ edges.

The absolute values above the $L_{1}$ edge as compared to the values between the $L_{3}$ and $L_{2}$ edges differ from the theoretical prediction.

These deviations are most clearly seen for Hf but are common for all atoms studied. Since the experimental data have been obtained with samples of different thicknesses, i.e., different absolute $\mathrm{x}$-ray attenuation (Table I) and mea-
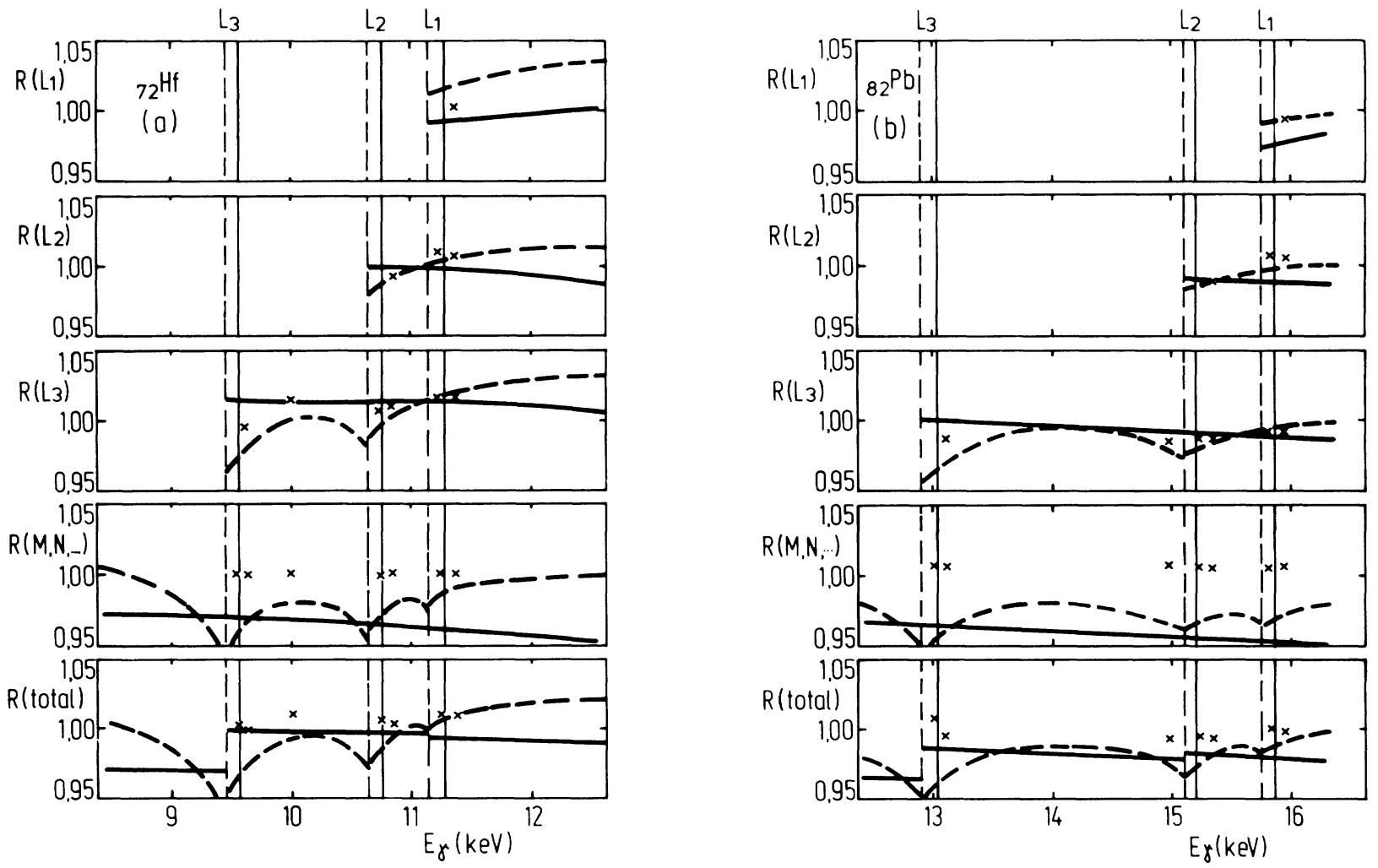

FIG. 3. Comparison of calculated photoelectric cross sections of (a) ${ }_{72} \mathrm{Hf}$ and $(\mathrm{b}){ }_{82} \mathrm{~Pb}$ for various subshells. For ease of comparison we have plotted the ratio $R$ of the various theoretical results and the corresponding fitted cross section [Eqs. (3) and (4)]. $\times$, RHS (Scofield, Ref. 15); _ _ IPA; - - , LRA (Liberman and Zangwill, Ref. 55). Vertical lines indicate $L$ edges; |, experimental energies, , energies as used in the IPA and LRA calculations. 
surements were made at different beam times (with the setup assembled new each time), we exclude a systematic experimental error. The deviations are unlikely to be due to solid-state effects since these are small for inner shells and an effect exhibiting the observed signature is not known. It can be ruled out that the deviations are caused by the scattering cross section since on the one hand this is small and on the other hand we observed the same type of deviations also for the induced x-ray fluorescence. ${ }^{38}$

Finally, we note that by normalization of the experimental data to theory at a different spectral region (i.e., above the $L_{1}$ edge) agreement within this region can be obtained: nevertheless, the deviations stated above remain.

\section{ELECTRON CORRELATIONS}

The observed deviations are not expected within the framework of a single-electron picture where an electron makes a transition from a bound state to the continuum in a frozen potential. This picture is a simplification: Photoionization also has some collective aspects, because the response of an atom to an external electromagnetic field is determined by the response of all electrons (see, e.g., Refs. 39 and 40). Two effects may be important for the photoionization of intermediate and inner shells: relaxation and dielectric polarization, as will now be discussed (see, e.g., Refs. 8 and 41 ).

Relaxation. The creation of an inner-shell vacancy by photon absorption changes the effective potential seen by the residual electrons; these react by a corresponding rearrangement of electron shells. This relaxation changes the potential experienced by the outgoing photoelectron and, as a result, the ionization cross section is modified. A subtle kind of relaxation is the dynamical rearrangement where the created vacancy decays during the emission of the photoelectron; for inner-shell ionization the dynamical rearrangement is important in the immediate threshold regime only.

Dielectric polarization. The external radiation field acts on all electron shells and distorts the whole atomic charge cloud. The response of the electrons to the distortion produces a mean field which reflects the atomic dielectric properties. The induced polarization causes a positiondependent dielectric screening (or antiscreening) of the external field. ${ }^{42}$ As a result, photoabsorption cross sections of individual subshells are modified in a fundamental way. For inner shells, we expect the modification of cross sections to be similar to the $x$-ray dispersion. Indeed, a typical dispersion curve (see, e.g., Ref. 43) is similar to the observed deviations between our experimental data and the predictions of independent-particle theories (Fig. 2, lower parts). Apparently, the deviations are mainly due to the dielectric polarization.

A quantitative investigation of electron correlations in photoionization may be performed by various theoretical approaches: random-phase approximation with exchange, ${ }^{39,40,44-46}$ many-body perturbation theory, $47-49$ and the $R$-matrix theory, ${ }^{50-52} \mathrm{~A}$ recent approach to the dielectric response problem is a treatment based on the time-dependent local-density approximation (TDLDA). ${ }^{53,54}$ This approach treats intra- and inter-shell correlations $^{8}$ simultaneously. The local-density approximation (LDA) is not an "approximate" Hartree-Fock (HF) scheme since $\mathrm{HF}$ treats exchange exactly and neglects correlation, whereas the LDA treats both on an equal footing, albeit in a nontransparent fashion for an inhomogeneous system. ${ }^{53}$

We have performed calculations of the inner-shell photoelectric cross sections in TDLDA employing the computer code of Liberman and Zangwill. ${ }^{55}$ This program does relativistic self-consistent-field calculations for the wave functions and calculates the response to a weak external oscillating field (dynamical polarization) in linear approximation. One useful feature of the program is the option for the treatment of correlation effects: These effects may be omitted, yielding the so-called independentparticle approach (IPA), or included, yielding the socalled linear-response approach (LRA). The differences between the IPA and LRA results directly reflect the importance of correlation effects.

Figure 3 shows a comparison of theoretical cross sections calculated in the RHS, ${ }^{15}$ IPA, and LRA (Ref. 55) approximations. To facilitate comparison, the ratio $R$ of the calculated values and the corresponding fitted analytical cross section [Eqs. (3) and (4)] is plotted. The predictions of the various calculations agree in their absolute values within a few percent for all $L$ subshells. Minor differences between the RHS and IPA results are presumably due to differences in the employed relativistic wave functions. As expected, these two theoretical approximations give a smooth exponential dependence of the cross sections on primary energy. By contrast, the LRA results exhibit pronounced features, such as, e.g., a decrease of cross sections in the vicinity of the absorption edges. A decrease occurs not only for a particular subshell at its threshold, but also at the thresholds of the other subshells. Such a behavior is typical for polarization effects.

Quantitative comparison between the LRA predictions and experiment is hampered by the fact that the calculated threshold energies used in the LRA calculations are smaller than experiment ${ }^{56}$ by approximately $100-150 \mathrm{eV}$ (depending on element and $L$ subshell). A simple displacement of the energy scale may introduce systematic errors, as cross sections depend strongly on energy. Nevertheless, as a trial we have shifted the theoretical LRA and IPA values for $\mathrm{Hf}$ by $115 \mathrm{eV}$ in energy scale and by $-1.9 \%$ in absolute height in order to compare them with experiment (Fig. 4). As can be seen, the LRA gives a nice quantitative description (within less than $0.5 \%$ ) of the experimentally observed features between the $L_{3}$ and $L_{2}$ edges, as well as between the $L_{2}$ and $L_{1}$ edges; apparently, the LRA treats the dominant collective effects properly. However, in the vicinity of and above the $L_{1}$ edge, deviations in absolute height and energy dependence are recognized; the origin of these deviations is not clear. The behavior found for HF is typical for all investigated elements (compare Figs. 2 and 3).

A stringent experimental test of correlation effects would be a direct measurement of the ionization cross section of each individual subshell. This can be accomplished by detecting the photoelectron or by making use of the vacancy decay in which an x-ray or an Auger elec- 


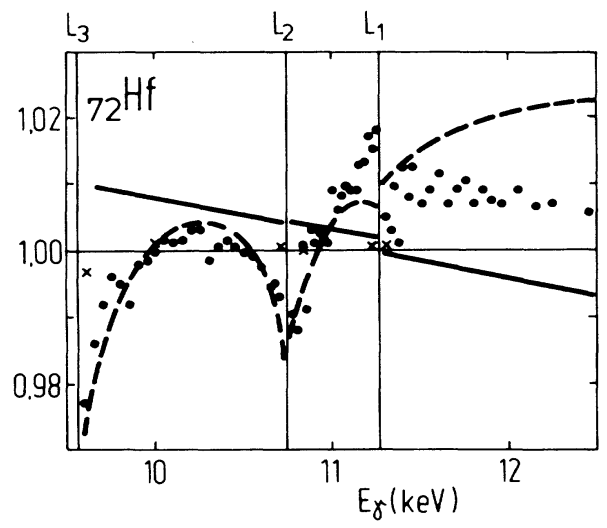

FIG. 4. Comparison of the total experimental and theoretical absorption cross section of ${ }_{72} \mathrm{Hf}$. To facilitate comparison, the ratio of the individual cross sections and the fitted cross section $\sigma_{\text {total }}^{\text {fit }}$ [Eqs. (3) and (4)] is plotted. e, experimental data (arbitrarily normalized); $\times$, RHS (Scofield, Ref. 15); - , IPA; - _ - , LRA (Liberman and Zangwill, Ref. 55; however, energy scale is shifted by $+115 \mathrm{eV}$ and absolute values are changed by $-1.9 \%$ to obtain better matching to the experimental data).

tron is emitted whose energy is specific for the ionized subshell. We have performed corresponding measurements of the spectrally resolved $x$-ray fluorescence. ${ }^{13,38}$ These measurements allow us to extract both subshell ionization cross sections and intrashell vacancy transfer by Coster-Kronig transitions. The fluorescence data clearly exhibit different slopes in the two regions between the $L_{3}$ and $L_{2}$ edges and between the $L_{2}$ and $L_{1}$ edges, similar to the behavior of the absorption cross sections. Evaluation of the measurements is in progress.

\section{CONCLUSION}

Photoionization of inner electron shells in heavy atoms is basically a one-electron process. Single-particle theories predict cross sections with an accuracy of a few percent. Collective effects are small and may be hard to identify. For example, previous work $^{16}$ seems to indicate an underestimate of the experimental ionization cross sections by Scofield's calculations ${ }^{15}$ in the energy regime from a few $\mathrm{keV}$ to about $20 \mathrm{keV}$ of the order of a few percent. Possibly this is due to the neglect of collective effects which increase the cross section here, as follows from comparative IPA and LRA calculations. ${ }^{57}$ In the present work we have measured the photoionization in the energy regime of the $L$ edges with high spectral resolution where collective effects exhibit characteristic energy-dependent features. By a careful experimental study and comparative calculations we were able to identify electroncorrelation effects here for the first time and to investigate them quantitatively. The features originate dominantly from the dielectric response (polarization) of electron shells to the external radiation field. The TDLDA theory by Liberman and Zangwill ${ }^{55}$ is able to reproduce the experimental total absorption cross section with about $\pm 1 \%$. This seems to be a quite satisfying situation both for scientific work on fundamental interactions and for applications in quantitative studies.

\section{ACKNOWLEDGMENTS}

The measurements at the Synchrotron Radiation Source in Daresbury were made successful by collaboration with Dr. G. N. Greaves. Professor Dr. H. O. Lutz made valuable suggestions on this work; K. Finck was engaged in the measurements. Financial support by the "Minister für Wissenschaft und Forschung des Landes NordrheinWestfalen" and the "Science and Engineering Research Council" is gratefully acknowledged.
*Former address: Fakultät für Physik, Universität Bielefeld, D-4800 Bielefeld, Germany.

${ }^{1}$ Atomic Inner-Shell Physics, edited by B. Crasemann (Plenum, New York, 1985).

${ }^{2}$ W. Jitschin, A. Kaschuba, H. Kleinpoppen, and H. O. Lutz, Z. Phys. A 304, 69 (1982).

${ }^{3}$ K. Finck, W. Jitschin, and H. O. Lutz, J. Phys. B 16, L409 (1983).

${ }^{4}$ W. Jitschin, Nucl. Instrum. Methods B4, 292 (1984).

${ }^{5}$ L. Sarkadi, J. Phys. B 19, 2519 (1986).

${ }^{6}$ A. F. Starace, in Fundamental Processes in Energetic Atomic Collisions, edited by H. O. Lutz, J. S. Briggs, and H. Kleinpoppen (Plenum, New York, 1983), p. 69.

${ }^{7}$ B. F. Sonntag, in Electronic and Atomic Collisions, edited by J. Eichler, I. V. Hertel, and N. Stolterfoht (North-Holland, Amsterdam, 1984), p. 459.

${ }^{8}$ M. Ya. Amusia, in $X-84$ Conference Proceedings, edited by A. Meisel and J. Finster (Karl-Marx-Universität, Leipzig, 1984), p. 33.

${ }^{9}$ H. Kleinpoppen, in Proceedings of the 30th Scottish Universi- ties Summer School in Physics, Aberdeen, 1985 (unpublished).

${ }^{10}$ M. H. Chen, in Atomic Inner-Shell Physics, edited by B. Crasemann (Plenum, New York, 1985), p. 31.

${ }^{11} \mathrm{~W}$. Mehlhorn, in Atomic Inner-Shell Physics, edited by B. Crasemann (Plenum, New York, 1985), p. 119.

${ }^{12}$ O. Keski-Rahkonen, G. Materlik, B. Sonntag, and J. Tulkki, J. Phys. B 17, L121 (1984).

${ }^{13}$ W. Jitschin, G. Materlik, U. Werner, and P. Funke, J. Phys. B 18, 1139 (1985).

${ }^{14}$ W. Jitschin, U. Werner, K. Finck, and H. O. Lutz, in HighEnergy Ion-Atom Collisions II, edited by D. Berényi and G. Hock (Elsevier, Amsterdam, 1985), p. 79.

15 J. H. Scofield, Lawrence Livermore Radiation Laboratory Report No. UCRL-51326 (1973) (unpublished).

${ }^{16} \mathrm{~J}$. H. Hubbell and Wm. J. Veigele, Comparison of Theoretical and Experimental Photoeffect Data $0.1 \mathrm{keV}$ to $1.5 \mathrm{MeV}$, Natl. Bur. Stand. (U.S.) Technical Note No. 901 (U.S. GPO, Washington, D.C., 1976).

${ }^{17}$ W. Jitschin, in Progress in Atomic Spectroscopy, Part D, edited by H. J. Beyer and H. Kleinpoppen (Plenum, New York, 
1987).

${ }^{18}$ R. Frahm, R. Haensel, W. Malzfeldt, W. Niemann, and P. Rabe, HASYLAB Jahresbericht 1982, p. 121 (unpublished).

${ }^{19}$ R. D. Deslattes, R. E. LaVilla, P. L. Cowan, and A. Henins, Phys. Rev. A 27, 923 (1983).

${ }^{20}$ International Tables for X-Ray Crystallography, edited by $\mathrm{K}$. Lonsdale (Kynoch, Birmingham, England, 1962), Vol. 3, p. 157.

${ }^{21}$ K. F. J. Heinrich, in The Electron Microprobe, edited by T. D. McKinley, K. F. J. Heinrich, and D. B. Wittry (Wiley, New York, 1966), p. 296.

${ }^{22}$ T. K. Kelly, Trans. Inst. Min. Metall. 75, B59 (1966).

${ }^{23}$ G. Materlik and V. O. Kostroun, Rev. Sci. Instrum. 51, 86 (1980).

${ }^{24}$ O. Keski-Rahkonen and M. O. Krause, At. Data 14, 139 (1974).

${ }^{25}$ G. S. Brown and S. Doniach, in Synchrotron Radiation Research, edited by H. Winick and S. Doniach (Plenum, New York, 1980), p. 353.

${ }^{26}$ D. R. Sandstrom and F. W. Lytle, Ann. Rev. Phys. Chem. 30, 215 (1979).

${ }^{27}$ P. Rabe, G. Tolkiehn, and A. Werner, J. Phys. C 12, 899 (1979).

${ }^{28}$ V. A. Biebesheimer, E. C. Marques, D. R. Sandstrom, F. W. Lytle, and R. B. Greegor, J. Chem. Phys. 81, 2599 (1984).

${ }^{29}$ F. Kohlrausch, Praktische Physik (Teubner, Stuttgart, 1968), in German.

${ }^{30}$ D. C. Creagh and J. H. Hubbell, X-Ray Spectrom. 11, ix (1982).

${ }^{31}$ J. H. Hubbell, Trans. Am. Nucl. Soc. 50, 153 (1985).

${ }^{32} \mathrm{Wm}$. J. Veigele, At. Data Tables 5, 51 (1973).

${ }^{33}$ J. H. Hubbell, Wm. J. Veigele, E. A. Briggs, R. T. Brown, D. T. Cromer, and R. J. Howerton, J. Phys. Chem. Ref. Data 4, 471 (1975).

${ }^{34} \mathrm{H}$. A. Bethe and E. E. Salpeter, Quantum Mechanics of Oneand Two-Electron Atoms (Plenum, New York, 1977).
${ }^{35}$ B. K. Agarwal, X-Ray Spectroscopy (Springer, Berlin, 1979).

36 J. H. Scofield (private communication).

${ }^{37}$ K. Zhang, E. A. Stern, and J. J. Rehr, in EXAFS and Near Edge Structure III, edited by K. O. Hodgson, B. Hedman, and J. E. Penner-Hahn (Springer, Berlin, 1984), p. 18.

${ }^{38}$ W. Jitschin and U. Werner, J. Vac. Sci. Technol. A (to be published).

${ }^{39}$ M. Ya. Amusia and N. A. Cherepkov, Case Stud. At. Phys. 5, 47 (1975).

${ }^{40}$ M. Ya. Amusia, Comments Atom. Mol. Phys. 8, 61 (1979).

${ }^{41}$ A. Zangwill and D. A. Liberman, J. Phys. B 17, L253 (1984).

${ }^{42}$ A. Zangwill and P. Soven, Phys. Rev. A 21, 1561 (1980).

${ }^{43}$ L. G. Parratt and C. F. Hempstead, Phys. Rev. 94, 1593 (1954).

${ }^{44}$ G. Wendin, J. Phys. B 4, 1080 (1971).

${ }^{45} \mathrm{G}$. Wendin, in Vacuum Ultraviolet Radiation Physics, edited by E. E. Koch (Pergamon, Oxford, England, 1975), p. 225.

${ }^{46} \mathrm{G}$. Wendin, in Photoionization and Other Probes of ManyElectron Interactions, edited by F. J. Wuilleumier (Plenum, New York, 1976), p. 61.

${ }^{47}$ H. P. Kelly, Adv. Chem. Phys. 14, 129 (1969).

${ }^{48}$ H. P. Kelly and S. L. Carter, Phys. Scr. 21, 448 (1980).

${ }^{49}$ H. P. Kelly, in Atomic Physics 8, edited by I. Lindgren, A. Rosén, and S. Svanberg (Plenum, New York, 1983), p. 305.

${ }^{50} \mathrm{P}$. G. Burke and K. T. Taylor, J. Phys. B 8, 2620 (1975).

${ }^{51}$ P. G. Burke, in Electronic and Atomic Collisions, edited by G. Watel (North-Holland, Amsterdam, 1978), p. 201.

${ }^{52}$ W. D. Robb, in Electronic and Atomic Collisions, edited by G. Watel (North-Holland, Amsterdam, 1978), p. 231.

${ }^{53}$ A. Zangwill, in Atomic Physics 8, edited by I. Lindgren, A. Rosén, and S. Svanberg (Plenum, New York, 1983), p. 339.

${ }^{54} Z$. Levine and P. Soven, Phys. Rev. Lett. 50, 2074 (1983).

${ }^{55}$ D. A. Liberman and A. Zangwill, Comput. Phys. Commun. 32, 75 (1984).

56J. A. Bearden and A. F. Burr, Rev. Mod. Phys. 39, 125 (1967).

${ }^{57}$ G. D. Doolen (unpublished calculations). 